\title{
Est-il temps d'examiner notre façon de faire les choses?
}

\section{Par Scot Morris, docteur en optométrie}

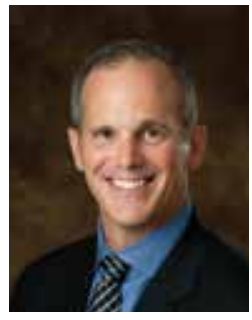

$D^{r}$ Scot Morris possède son propre cabinet à Denver, au Colorado. Il est aussi cofondateur de 4ECPs - une entreprise de ressources professionnelles pour les optométristes. 4ECPs englobe trois divisions : Emplois Formation - Marketing. Consultez www.4ECPs.com pour en savoir davantage. Scot est aussi rédacteur en chef de la publication Optometric Management, conférencier, auteur, consultant et innovateur en affaires.

$\mathrm{S}_{\mathrm{m}}^{\mathrm{o}}$ ouvent, dans notre quotidien, nous refaisons toujours la même chose. Disons que c'est la routine. Mais est-ce la meilleure façon et la façon la plus efficace de faire les choses, où est-ce simplement " notre façon de faire "? Envisageons les choses du point de vue du consommateur. Combien de temps devrait durer un examen de la vue? Quinze minutes, trente minutes, quarante-cinq? Pour chacun d'entre nous, le temps est la seule chose dont nous n'avons jamais trop; il est impossible à récupérer, et c'est notre ressource la plus précieuse. Accordons-nous au temps de nos clients et à notre temps la valeur qu'il mérite?

Je mets au défi chacun d'entre vous qui me lisez de travailler une heure de moins par jour. C'est exact, une heure de moins. Que feriez-vous de cette heure? J'ai votre attention maintenant?

Apprenons maintenant à "trouver " cette heure. Si vous recevez 15 patients par jour et que vous pouvez " gagner» quatre minutes par patient, vous aurez alors une heure supplémentaire. Quatre petites minutes! Dans la plupart des cas, les cabinets pourraient améliorer leur efficacité de 20 à $40 \%$ (8 à 12 minutes) et améliorer considérablement leur bilan simplement en analysant ce que fait réellement chaque personne au bureau tous les jours. Ce processus s'appelle l'analyse de l'efficacité du déroulement du travail.

Toutes les entreprises ont un processus de déroulement du travail. De nombreuses entreprises n'ont cependant aucune idée de leur processus. Il y a environ 102 étapes entre la prise de rendez-vous par un patient et le rendez-vous ultérieur l'année suivante, et c'est sans compter le marketing, les ressources humaines, la gestion des stocks ou les tâches opérationnelles. Chacune de ces étapes est une occasion saisie ou perdue d'éduquer votre client ou de vendre quelque chose, et une occasion de faire bonne ou mauvaise impression. Si vous voulez vraiment fournir de meilleurs soins, donner un service plus efficace et améliorer votre bilan, cet exercice sera l'une des expériences les plus révélatrices et bénéfiques que vous entreprendrez à titre d'homme ou de femme d'affaires. En remplaçant le raisonnement médical par le raisonnement commercial, vous commencerez à penser au fonctionnement de chaque étape du processus.

Comment pouvons-nous améliorer à la fois notre efficience et notre efficacité?

Cela est possible en quatre étapes.

PREMIËRE ÉTAPE : Déterminez où vous en êtes. Combien de temps dure une consultation avec un patient actuellement? Nous dirons le «porte-à-porte » (de l'arrivée du patient à la porte du bureau de l'optométriste jusqu'à son départ après l'examen). Pour commencer, notez le temps que vous croyez qu'il vous faut. Appelons ça l'estimation. Déterminez maintenant la durée « réelle » de la consultation. Notez l'heure d'arrivée du patient et l'heure à laquelle l'optométriste a terminé son travail (le moment où le client passe à la partie vente de la consultation). Déterminez ensuite vos ratios réels. La meilleure façon de les évaluer consiste à mesurer le nombre de consultations par heure. Vous y arriverez en prenant le nombre total de patients divisé par les heures travaillées dans une période donnée.

DEUXIÈME ÉTAPE : Déterminez ce que vous faites. L'étape suivante consiste à définir chacune de la centaine d'étapes de l'expérience des consommateurs. Déterminez les processus applicables à chaque étape de l'expérience du consommateur à votre cabinet. Commencez lentement. Demandez à toutes les personnes qui travaillent à votre cabinet de dresser une liste précise de chaque étape effectuée par votre bureau de réception. Demandez ensuite à toutes ces personnes de placer les étapes dans l'ordre de leur déroulement. Demandez-leur de faire l'exercice individuellement. Faites-le également 
vous-même. Puis recueillez et combinez toutes les réponses. Préparez-vous à être étonné. Ne soyez pas surpris si chacun donne des réponses complètement différentes. Le diagramme ci après donne un exemple des diverses étapes qui peuvent constituer le simple processus de "rendez-vous et accueil ». Voyez cela comme un défi visant à mettre tout le monde au diapason. Ce processus prendra quelques semaines. Il vous faudra six à huit semaines pour l'achever.

TROISIÈME ÉTAPE : Rationalisez les processus. Analysez maintenant chaque étape; ce qu'on y fait et ce qu'on y dit. Si un processus ne sert pas à éduquer, à vendre ou à améliorer l'efficience, éliminez-le. Répétez ensuite cette étape pour chaque service (test préalable, fournisseur, opticien, lentilles de contact, départ, facturation). Déterminez qui dit quoi. Qui fait quoi. Que font-ils ou que disent-ils? À qui le disent-ils?
QUATRIÈME ÉTAPE : Faites l'examen des communications et affinez les processus (textes et protocoles). Demandez aux membres de votre personnel d'écrire ce qu'ils disent aux consommateurs à chaque étape. S'ils ne font ni éducation ni vente, vous devez revoir ce qu'ils disent. Décidez ensuite si chaque étape pourrait être faite de façon plus efficiente ou carrément être éliminée. Ce processus pourrait prendre environ un mois. Après cette étape, passez aux étapes suivantes: test préalable, examen de l'optométriste, opticien, lentilles de contact, départ, et facturation. Ce processus prend du temps, mais les transformations radicales qu'il entraîne en matière d'efficience et de rentabilité en valent vraiment la peine. À chaque point du processus, nous devons essentiellement décider comment sont présentés les éléments suivants. Examinez chacun des points du tableau à droite lorsque vous évaluez les choses à dire à chaque étape.

\section{Considérations liées à la communication}

1. Que tentons-nous d'accomplir? (Notre but)

2. Que tentons-nous de vendre ou de promouvoir?

3. Quels sont leurs besoins ou leurs souhaits? Comment pouvons-nous le déterminer?

4. Comment pouvons-nous les orienter vers les sujets dont nous voulons parler?

5. Quels sont les obstacles potentiels à la communication?

6. Est-ce vraiment ce que nous disons?

7. Sommes-nous compris? Comment le savez-vous?

8. Comment pouvons-nous assurer un suivi? 